\title{
Perfil clínico y epidemiológico de los pacientes con diagnóstico de neurocisticercosis en dos hospitales de Chiclayo, Perú
}

\author{
Giuseppe Rojas-Panta ${ }^{1}$, Carlos J. Toro-Huamanchumo ${ }^{1}$, Enrique Altamirano-Mego ${ }^{2}$
}

RESUMEN

Objetivo: Describir el perfil clínico y epidemiológico de los pacientes con diagnóstico de neurocisticercosis en dos hospitales de Chiclayo, Perú.

Materiales y métodos: Estudio descriptivo, transversal y retrospectivo, realizado en los hospitales Almanzor Aguinaga Asenjo y Regional Lambayeque. Se revisaron 96 historias clínicas de pacientes con diagnóstico de neurocisticercosis atendidos en el año 2014.

Resultados: La media de edad de los pacientes fue 42,8 4 18,8 años, $52,1 \%$ fue de sexo femenino, $26 \%$ provenía de Chiclayo y $54,2 \%$ recibieron atención por primera vez. El síntoma de consulta más frecuente fue la cefalea, y a todos se les realizó algún método de diagnóstico por imágenes. La ubicación fue en su mayoría cerebral y el estadio más común fue el calcificado. El tratamiento más utilizado fueron los anticonvulsivantes y los analgésicos.

Conclusiones: La neurocisticercosis afecta principalmente a la población económicamente activa, es más frecuente en mujeres y la manifestación clínica más común es la cefalea.

Palabras clave: Neurocisticercosis; epidemiología; taenia solium; Perú.

\section{Clinical and epidemiological profile of patients diagnosed with neurocysticercosis at two hospitals in Chiclayo, Peru}

ABSTRACT

Objective: To describe the clinical and epidemiological profile of patients diagnosed with neurocysticercosis at two hospitals in Chiclayo, Peru.

Materials and methods: A descriptive, cross-sectional and retrospective study was performed at the Almanzor Aguinaga Asenjo Hospital and Lambayeque Regional Hospital. Ninety-six (96) medical records of patients attended in 2014 with a diagnosis of neurocysticercosis were reviewed.

Results: The mean age of the patients was $42.8 \pm 18.8$ years old, $52.1 \%$ were female, $26 \%$ came from Chiclayo and $54.2 \%$ received care for the first time. The most common symptom leading to medical consultation was headache. All patients underwent a diagnostic imaging method. The location of the parasite was mostly in the brain and the calcified stage was the most common one. The most widely used treatments were anticonvulsants and pain relievers.

Conclusions: Neurocysticercosis mainly affects the economically active population, is more common in women and the most common clinical manifestation is headache.

Keywords: Neurocysticercosis; epidemiology; taenia solium; Peru.

1. Facultad de Medicina Humana, Universidad de San Martín de Porres. Chiclayo, Perú.

2. Servicio de Neurología, Hospital Regional Lambayeque. Chiclayo, Perú. 


\section{INTRODUCCIÓN}

La neurocisticercosis (NCC) es una enfermedad endémica en el Perú y es la parasitosis más frecuente del sistema nervioso central (SNC). La prevalencia de NCC no se conoce pero la de teniasis es de alrededor $1 \%$ en la población. Además, la prevalencia de epilepsia es de 15/1000 habitantes, de los cuales $30-50 \%$ están de alguna manera asociados a NCC ${ }^{(1)}$. En Lambayeque, la sero-prevalencia contra Taenia solium es de $22,8 \%$, lo cual significa que esta población ha estado expuesta a este agente alguna vez en su vida ${ }^{(1,2)}$.

Se encuentra principalmente en zonas alto andinas y en menor medida en la costa y selva. Las comunidades rurales presentan una alta prevalencia de teniasis y cisticercosis, alcanzando un $17 \%$ y $75 \%$ en humanos y porcinos, respectivamente ${ }^{(3,4)}$. Ello se ve favorecido por factores como la pobreza, escasa educación sanitaria, falta de higiene, deficiencias en eliminación de excretas y residuos sólidos al aire libre; así como el consumo de agua no potable, crianza y beneficio clandestino de cerdos y el consumo de esta carne sin inspección veterinaria ${ }^{(5)}$.

El hombre es el único huésped definitivo de Taenia solium, sin embargo, también puede ser huésped intermediario. Se contagia al ingerir los cisticercos presentes en la carne de cerdo y desarrolla teniasis, forma intestinal de la enfermedad. Cuando es huésped intermediario, el hombre ingiere los huevos del parásito, los cuales migran hacia los tejidos, mayormente al SNC ${ }^{(6)}$.

El cuadro clínico depende de la localización, tamaño y número de quistes a nivel del encéfalo. Para el diagnóstico se emplean técnicas imagenológicas y serológicas. Entre las primeras se encuentran la Tomografía Axial Computarizada (TAC) y la Resonancia Magnética (RM), mientras que el Western Blot (WB) es la prueba de elección para el diagnóstico serológico. El tratamiento es por lo general médico, siendo quirúrgico en determinados casos. El tratamiento médico se basa en el uso de cisticidas, anticonvulsivantes, glucocorticoides, etc. ${ }^{(7)}$.

Un estudio previo realizado en un hospital peruano encontró que el grupo etario más afectado fue la población económicamente activa y la clínica más frecuente fue la cefalea que no cedía al tratamiento ${ }^{(8)}$. A nivel de Lambayeque no se han encontrado estudios publicados que aborden la clínica y epidemiología de la NCC.

El objetivo de este trabajo fue describir el perfil clínico y epidemiológico de los pacientes con diagnóstico de neurocisticercosis en dos hospitales de Chiclayo, Perú, durante el año 2014.

\section{MATERIALES Y MÉTODOS}

\section{Diseño del estudio y contexto}

Estudio descriptivo, transversal y retrospectivo, realizado en los hospitales Almanzor Aguinaga Asenjo y Regional
Lambayeque en Chiclayo. La información fue proporcionada por la Oficina de Inteligencia Sanitaria del Hospital Almanzor Aguinaga Asenjo y por la Oficina de Estadística e Informática del Hospital Regional Lambayeque.

\section{Población de estudio y procedimiento}

Se revisaron 96 historias clínicas de pacientes con diagnóstico de NCC atendidos en el año 2014, considerándose sólo a los pacientes con diagnóstico confirmado de NCC de los Servicios de Neurología, Medicina Interna, Pediatría, Infectología y Geriatría. Se excluyeron las historias clínicas que estuvieron ilegibles u omitían datos importantes para este estudio.

Se recolectaron los siguientes datos: Número de historia clínica, edad, sexo, lugar de procedencia; condición de atención en el hospital, síntomas de consulta, método diagnóstico empleado, ubicación anatómica del parásito, estadio de evolución y tratamiento prescrito.

\section{Análisis estadístico}

La información obtenida a través de la ficha de recolección de datos se transfirió a una base de datos en Microsoft Excel 2010 con doble digitación. Posterior al control de calidad, se ingresó la base al paquete estadístico STATA v13.0 para su análisis. Se empleó estadística descriptiva previa evaluación de los supuestos de normalidad usando Shapiro Wilk, de acuerdo a eso se empleó media y desviación estándar para la edad y mediana y rangos intercuartílicos para el número de signos y síntomas. Las variables categóricas se presentaron con frecuencias y porcentajes. Para el cruce entre variables cualitativas politómicas y cuantitativas, se usó la prueba de ANOVA de un factor y la prueba de Kruskal Wallis, según la normalidad de la variable cuantitativa. Para las variables categóricas se usó el test exacto de Fisher.

\section{Aspectos éticos}

El estudio fue aprobado por el Comité de Ética en Investigación del Hospital Regional Lambayeque. Después de recolectar los datos de cada paciente, los números de las historias clínicas fueron eliminados de la base de datos, con lo cual quedó una lista anónima y se usaron códigos individuales para el análisis.

\section{RESULTADOS}

Las 96 historias clínicas revisadas tuvieron diagnóstico definitivo de neurocisticercosis. La media de edad de los pacientes fue $42,8 \pm 18,8$ años. El $52,1 \%$ fue de sexo femenino y la mayoría de pacientes procedía de lugares diferentes del distrito de Chiclayo (Tabla 1). 
Tabla 1. Características sociodemográficas de los pacientes con diagnóstico definitivo de neurocisticercosis

\begin{tabular}{|l|c|c|}
\hline \multicolumn{1}{|c|}{ Característica } & $\mathbf{n}$ & $\%$ \\
\hline Sexo & & \\
\hline Femenino & 50 & 52,1 \\
\hline Masculino & 46 & 47,9 \\
\hline Procedencia & & \\
\hline Chiclayo & 25 & 26,0 \\
\hline Jaén & 7 & 7,3 \\
\hline Otros & 64 & 66,7 \\
\hline Condición & & \\
\hline Nuevo & 52 & 54,2 \\
\hline Control & 44 & 45,8 \\
\hline
\end{tabular}

El síntoma de consulta más prevalente fue la cefalea (77\%), seguida por epilepsia (48,9\%). La ubicación del parásito fue en su mayoría parenquimal y dentro de ellas la cerebral $(87,5 \%)$. De los estadios del parásito, el calcificado fue el más frecuente con $46,2 \%$ (Tabla 2 ).

Tabla 2. Características clínicas y epidemiológicas de los pacientes con diagnóstico definitivo de neurocisticercosis

\begin{tabular}{|l|c|c|}
\hline \multicolumn{1}{|c|}{ Característica } & $\mathbf{n}$ & $\%$ \\
\hline Motivo de consulta & 74 & 77,0 \\
\hline Cefalea & 47 & 48,9 \\
\hline Crisis epiléptica & 21 & 21,8 \\
\hline Náuseas o vómitos & 15 & 15,6 \\
\hline Mareos & 9 & 9,3 \\
\hline Problemas visuales & 7 & 7,2 \\
\hline Pérdida de conciencia & 7 & 7,2 \\
\hline Dificultad en la marcha & 13 & 13,4 \\
\hline Otros* & & \\
\hline Ubicación del parásito & 84 & 87,5 \\
\hline Cerebral & 5 & 5,2 \\
\hline Cerebeloso & 3 & 3,1 \\
\hline Cuarto ventrículo & 2 & 2,1 \\
\hline Subaracnoideo & 1 & 1,0 \\
\hline Tronco encefálico & 1 & 1,0 \\
\hline Tercer ventrículo & & \\
\hline Estadio del parásito & 43 & 46,2 \\
\hline Calcificado & 28 & 30,1 \\
\hline Vesicular & 13 & 14,0 \\
\hline Coloidal & 9 & 9,7 \\
\hline Nodular & & \\
\hline
\end{tabular}

*Otros: Relajación de esfínteres, parestesias, paresias, parálisis facial, somnolencia, etc.

A todos los pacientes se les realizó al menos un método de diagnóstico por imágenes (TAC o RM). La distribución de los fármacos o tratamientos usados se presentan en la (Tabla 3). 
Perfil clínico y epidemiológico de los pacientes con diagnóstico de neurocisticercosis en dos hospitales de Chiclayo, Perú

Tabla 3. Diagnóstico y tratamiento de los casos hospitalizados por neurocisticercosis

\begin{tabular}{|l|c|c|}
\hline \multicolumn{1}{|c|}{ Variable } & $\mathbf{n}$ & $\%$ \\
\hline Método diagnóstico & & \\
\hline TAC & 45 & 46,9 \\
\hline RM & 5 & 5,2 \\
\hline TAC+RM & 19 & 19,8 \\
\hline TAC+WB & 12 & 12,5 \\
\hline RM+WB & 2 & 2,1 \\
\hline TAC+RM+WB & 13 & 13,5 \\
\hline Tratamiento utilizado & & \\
\hline Anticonvulsivantes & 73 & 76,0 \\
\hline Analgésicos & 73 & 76,0 \\
\hline Glucocorticoide & 46 & 47,9 \\
\hline Albendazol & 40 & 41,7 \\
\hline Cirugía & 5 & 5,2 \\
\hline Praziquantel & 2 & 2,1 \\
\hline Otros* & 53 & 55,2 \\
\hline
\end{tabular}

Se encontraron diferencias en el estadio del parásito según el sexo de los pacientes $(p<0,001 ;$ ANOVA). El número de signos y síntomas presentados tuvo una mediana igual a 2, cifra que se modificó según el estadio del parásito $(p<0,05$; Kruskal Wallis), también se encontraron diferencias estadísticas en la condición del paciente y el método diagnóstico (Tabla 4).

Tabla 4. Características sociodemográficas y clínicas según el estadio del parásito

\begin{tabular}{|c|c|c|c|c|c|}
\hline \multicolumn{6}{|c|}{ Estadio } \\
\hline Característica & Vesicular & Coloidal & Nodular & Calcificado & $\mathbf{p}^{*}$ \\
\hline \multicolumn{6}{|l|}{ Sexo } \\
\hline Femenino & 14 & 7 & 4 & 24 & \multirow[t]{2}{*}{$>0,05$} \\
\hline Masculino & 14 & 6 & 5 & 19 & \\
\hline \multicolumn{6}{|l|}{ Condición } \\
\hline Nuevo & 27 & 11 & 4 & 7 & \multirow[t]{2}{*}{$<0,001$} \\
\hline Control & 1 & 2 & 5 & 36 & \\
\hline \multicolumn{6}{|c|}{ Método Diagnóstico } \\
\hline TAC & 7 & 2 & 4 & 32 & \multirow[t]{6}{*}{$<0,001$} \\
\hline RM & 2 & 1 & 1 & 1 & \\
\hline $\mathrm{TAC}+\mathrm{RM}$ & 5 & 4 & 1 & 7 & \\
\hline TAC + WB & 5 & 2 & 2 & 3 & \\
\hline$R M+W B$ & 1 & 0 & 1 & 0 & \\
\hline$T A C+R M+W B$ & 8 & 4 & 0 & 0 & \\
\hline
\end{tabular}

*Prueba exacta de Fisher 


\section{DISCUSIÓN}

De los datos de pacientes analizados se encontró que la distribución por sexo coincide con lo reportado en la literatura internacional, siendo el sexo femenino el más frecuente ${ }^{(9,10)}$. Esto puede deberse a que en zonas consideradas endémicas las personas dedicadas a labor doméstica (cocineras y amas de casa) suelen estar más expuestas (11). La mayoría de pacientes estuvo en el intervalo de edad de la población económicamente activa (15-65 años), afectando en alguna medida sus ingresos, al ser hospitalizados, comprar medicación, etc. ${ }^{(12)}$.

La mayoría de pacientes provenía de distritos alejados de Chiclayo (de los departamentos de Cajamarca, Amazonas y algunos de Lambayeque). Es probable que en estas zonas la crianza y consumo de cerdo sea deficiente, lo cual sumado a malas condiciones higiénicas y de saneamiento favorecen la transmisión de esta parasitosis ${ }^{(1)}$.

Está descrito que la causa más frecuente de epilepsia en países en vías de desarrollo es la neurocisticercosis ${ }^{(1)}$, siendo además el motivo de consulta más frecuente reportado en la literatura ${ }^{(7,13,14)}$. Sin embargo, en este estudio la principal manifestación clínica fue la cefalea, lo que concuerda con un estudio realizado en Perú por Esquicha et al ${ }^{(8)}$.

Todos los pacientes fueron estudiados con algún método de diagnóstico por imágenes (TAC o RM), que por sí solos permiten hacer el diagnóstico en muchos de los casos ${ }^{7,15)}$. En algunos de ellos se requirió también la prueba de Western Blot (la cual es confirmatoria) o las tres pruebas juntas, posiblemente debido a una duda diagnóstica por otro proceso que curse con hallazgos radiográficos y clínicos similares, como por ejemplo la hidatidosis cerebral (aunque rara), alguna neoplasia cerebral, accidente cerebro vascular (ACV), absceso cerebral, etc. ${ }^{(7)}$.

Se conoce que la ubicación principal, la cual origina las manifestaciones clínicas típicas, es la cerebral (7). Entre las otras manifestaciones encontradas estuvieron las alteraciones visuales, la dificultad de la marcha y los desórdenes psiquiátricos. Las alteraciones visuales se deben a compresión mecánica del nervio óptico o el quiasma óptico por el cisticerco o por la presencia de este en el lóbulo occipital, pudiendo manifestarse como anopsia, hemianopsia o pérdida de la agudeza visual. La dificultad de la marcha es consecuencia de la paresia de los miembros inferiores por la presencia del parásito en la corteza del lóbulo frontal y la reacción inmune que se origina, también se describe que la presencia del cisticerco en territorio cercano a arterias de gran calibre como a pequeños vasos intracerebrales podría dar lugar a la misma clínica debido a isquemia. Los desórdenes psiquiátricos atribuidos a NCC incluye demencia, depresión, trastorno psicóticos y alteración cognitiva ${ }^{(13,16,17)}$.

El estadio del parásito hallado con más frecuencia al momento de la atención fue el calcificado, lo cual concuerda con lo referido en la bibliografía, que menciona que la forma calcificada puede encontrarse hasta en 10 a $20 \%$ de la población en zonas endémicas ${ }^{(15)}$. Se encontró diferencia estadística entre la condición del paciente al momento de la atención en el hospital (nuevo o control) y los estadios del parásito, estando la mayoría de pacientes nuevos en los estadios vesicular, coloidal y nodular al momento de la atención en el hospital. Esto se debe a que en estos estadios las manifestaciones clínicas iniciales se presentan comúnmente ${ }^{17,15)}$ y llevan a una primera búsqueda de atención médica.

Pocos pacientes tuvieron el estadio calcificado del parásito y acudían por primera vez. Está descrito el inicio de manifestaciones clínicas en este estadio, ya que posiblemente fueron asintomáticos o tuvieron molestias mínimas en los estadios anteriores. Este hecho ocurre más a menudo en países considerados endémicos para la enfermedad ${ }^{(10,15)}$. El número de síntomas fue significativamente más frecuente en el estadio coloidal. Esto puede deberse a que la intensidad del cuadro clínico es mayor en los tres primeros estadios, siendo el coloidal el que reporta mayor número de manifestaciones clínicas y complicaciones asociadas a la gravedad.

Este hecho está relacionado a la degeneración de parásito, lo cual desencadena la reacción inflamatoria que da origen a las manifestaciones clínicas ${ }^{(17,18)}$.

Se encontró que a mayor edad del paciente, más avanzado fue el estadio del parásito. Esto se debe a que una vez producida la infección y se desarrolla el estadio vesicular, este sigue un curso evolutivo inevitable hasta que se degenera por completo y llega al estadio calcificado ${ }^{(19)}$. Desde que se produce la infección hasta la aparición de los manifestaciones clínicas transcurre un tiempo de 3 a 10 años en promedio, siendo este lapso muy variable ${ }^{(20,21)}$.

En nuestro estudio no encontramos diferencias estadísticas entre el sexo del paciente y el estadio del parásito, lo cual difiere con lo reportado en literatura. Se describe que las mujeres presentan mayor reacción inmunológica frente al parásito ${ }^{(10)}$, mayor edema perilesional (estadios coloidal y nodular) y por lo tanto mayor severidad del cuadro en comparación con los varones, en quienes el estadio vesicular es el descrito con mayor frecuencia ${ }^{(10,22)}$.

En el estadio vesicular la mayor parte de pacientes requirió dos o más pruebas diagnósticas (TAC, RM y Western Blot) para establecer el diagnóstico. Si bien la TAC tiene una alta sensibilidad y especificidad para el diagnóstico de NCC ${ }^{(15)}$, la RM ofrece mayores detalles de la imagen especialmente en la lesiones con realce, la ubicación en la fosa posterior y ventricular. La prueba Western Blot si bien puede dar el diagnóstico definitivo, un resultado negativo no descarta la enfermedad ${ }^{(15,23)}$. A diferencia del estadio vesicular, en el calcificado se encontró que la mayoría solo requirió la TAC como método de ayuda diagnóstica, lo cual se debe a que es el examen de elección para visualizar estructuras calcificadas ${ }^{(15)}$. En los estadios coloidal y nodular el uso de la RM fue mayor, ya que en estos casos este examen aporta más detalles de la lesión con realce.

En cuanto al tratamiento, se encontró que aproximadamente en tres cuartos de los casos se indicaron 
fármacos antiepilépticos y analgésicos. Esto se explica por recomendaciones actuales de dar anticonvulsivantes a todos los pacientes con enfermedad en los tres primeros estadios; incluso si el motivo de consulta no es epilepsia, con el fin de prevenir un evento epiléptico que pueda darse al momento de dar cisticidas o en el transcurso de degeneración del cisticerco. En el estadio calcificado se indican anticonvulsivantes solo si el paciente persiste con crisis epilépticas ${ }^{(7,24)}$. Los analgésicos fueron indicados en pacientes con cefalea y en los que fueron intervenidos quirúrgicamente. El cisticida de uso más frecuente fue el albendazol, el cual se dio en similar porcentaje de casos que los glucocorticoides. Su uso en conjunto se da con el fin de evitar una respuesta inmune exagerada al morir el cisticerco, la cual empeoraría el cuadro clínico del paciente y habría mayor daño tisular ${ }^{(24)}$.

La cirugía fue necesaria en 5 pacientes, 4 de ellos tenían una ubicación ventricular del parásito, se les realizó derivación ventrículo-peritoneal a todos ellos. El caso restante fue intervenido por hipertensión endocraneana, siendo sometido a craneotomía descompresiva.

Una de las limitaciones de este estudio es que se evaluó pacientes solo con caso confirmado en las sedes donde se realizó el estudio, no considerando la población que acudió a otros hospitales o no usó los servicios de salud. Sin embargo, se cubrió la mayoría de pacientes ya que las sedes incluidas son las de referencia en la región. Por otra parte, al ser un estudio de fuentes secundarias, quizá algunas variables de interés no fueron incluidas. Se recomienda que estudios posteriores profundicen y analicen los factores asociados a las manifestaciones clínicas, complicaciones, abordaje quirúrgico, recidivas, entre otros.

En conclusión, la neurocisticercosis afecta principalmente a la población económicamente activa, es más frecuente en mujeres y la manifestación clínica más común es la cefalea.

Recalcamos la importancia del conocimiento de la fisiopatología, clínica y tratamiento para el adecuado diagnóstico y tratamiento de esta enfermedad endémica en nuestro país, así como el conocimiento de la biología y ciclo de vida del parásito con la finalidad de establecer y mejorar las medidas de control.

\section{REFERENCIAS BIBLIOGRÁFICAS}

1. Garcia HH, Gonzalez AE, Rodriguez S, Gonzalvez G, LlanosZavalaga F, Tsang VC, et al. Epidemiology and control of cysticercosis in Peru. Rev Peru Med Exp Salud Publica. 2010;27(4):592-7.

2. Escalante H, Pereda F, Sánchez M, Schulz H, Torres P, Davelois K. Pacientes neurológicos Del noroeste Del Perú con serología positiva por western blot a la larva de Taenia solium. Rev Peru Med Exp Salud Publica. 2004;21(2):92-7.

3. Cysticercosis Working Group in Peru. The marketing of cysticercotic pigs in the Sierra of Peru. The Cysticercosis Working Group in Peru. Bull World Health Organ. 1993;71(2):223-8.
4. Ministerio de Salud. Teniasis / cisticercosis por taenia solium un serio problema de Salud Pública en el Perú [Internet]; 2001 [Citado el 12 de abril de 2015]. Disponible en: http://www. dge.gob.pe/publicaciones/pub_invepi/iepi0.pdf

5. Murrell KD. WHO/FAO/OIE Guidelines for the surveillance, prevention and control of taeniosis/cysticercosis. Paris: OIE; 2005. 1-139. Disponible en: http://www.oie.int/doc/ged/ d11245.pdf

6. Del Brutto 0 . Neurocisticercosis: actualización en diagnóstico y tratamiento. Neurologia. 2005;20(8):412-8.

7. Sinha S, Sharma BS. Neurocysticercosis: a review of current status and management. J Clin Neurosci Off J Neurosurg Soc Australas. 2009;16(7):867-76.

8. Esquicha JA, Falcón N, Oshiro S. Características clínicas y epidemiológicas de los pacientes con neurocisticercosis en un hospital general de Lima. Rev Medica Hered. 2012;23(1):4-10.

9. Fleury A, Dessein A, Preux PM, Dumas M, Tapia G, Larralde $C$, et al. Symptomatic human neurocysticercosis--age, sex and exposure factors relating with disease heterogeneity. J Neurol. 2004;251(7):830-7.

10. Fleury A, Escobar A, Fragoso G, Sciutto E, Larralde C. Clinical heterogeneity of human neurocysticercosis results from complex interactions among parasite, host and environmental factors. Trans R Soc Trop Med Hyg. 2010;104(4):243-50.

11. Garcia HH, Gilman RH, Gonzalez AE, Pacheco R, Verastegui $M$, Tsang VCW. Human and porcine Taenia solium infection in a village in the highlands of Cusco, Peru. Acta Trop. 1999;73(1):31-6.

12. Rajkotia Y, Lescano AG, Gilman RH, Cornejo C, Garcia HH, Cysticercosis Working Group of Peru. Economic burden of neurocysticercosis: results from Peru. Trans R Soc Trop Med Hyg. 2007;101(8):840-6.

13. Carpio A. Clinical Diagnoses of Neurocysticercosis. En: Foyaca Sibat $\mathrm{H}$, editor. Novel Aspects on Cysticercosis and Neurocysticercosis [Internet]. InTech; 2013 [citado el 12 de octubre de 2015]. Disponible en: http://www. intechopen.com/books/novel-aspects-on-cysticercosis-andneurocysticercosis/clinical-diagnoses-of-neurocysticercosis

14. Escalante C, Escalante G. Neurocisticercosis en el niño. Análisis epidemiológico y clínico. Rev Neuropsiquiatr. 2013;68(3-4):201-11.

15. Kimura-Hayama ET, Higuera JA, Corona-Cedillo R, Chávez-Macías L, Perochena A, Quiroz-Rojas LY, et al. Neurocysticercosis: radiologic-pathologic correlation. Radiogr Rev Publ Radiol Soc N Am Inc. 2010;30(6):1705-19.

16. Saavedra H, Gonzales I, Alvarado MA, Porras MA, Vargas V, Cjuno RA, et al. Diagnóstico y manejo de la neurocisticercosis en el Perú. Rev Peru Med Exp Salud Pública. 2010;27(4):58691.

17. Del Brutto O, Garcia HH. Clinical Manifestations of Neurocysticercosis. En: Cysticercosis of the Human Nervous System. 1a ed. Berlin: Springer; 2014.

18. Del Brutto O, Garcia HH. Neuropathology of Cysticercosis. En: Cysticercosis of the Human Nervous System. 1a ed. Berlin: Springer; 2014.

19. Valenzuela-Borrayo LC. Neurocisticercosis en México, revisión de la literatura. Hipoc Rev Med. 2010;23:4-8.

20. The center for food security and public health. Taenia infections. Tech Factsheets [Internet]; 2005. Disponible en: http://www.cfsph.iastate.edu/Factsheets/pdfs/taenia.pdf

21. Del Brutto O, Garcia HH. Inmunopathology of Taeniasis and Cysticercosis. En: Cysticercosis of the Human Nervous System. 1a ed. Berlin: Springer; 2014. 
22. Del Brutto O, Garcia HH. Epidemiology of Human Cysticercosis in Endemic Regions. En: Cysticercosis of the Human Nervous System. 1a ed. Berlin: Springer; 2014.

23. Del Brutto O, Garcia HH. Diagnosis of Taeniasis and Cysticercosis. En: Cysticercosis of the Human Nervous System. 1a ed. Berlin: Springer; 2014.

24. Del Brutto O, Garcia HH. Management of Neurocysticercosis. En: Cysticercosis of the Human Nervous System. 1a ed. Berlin: Springer; 2014.
Fuentes de financiamiento:

Este artículo ha sido financiado por los autores.

Conflictos de interés:

Los autores declaran no tener ningún conflicto de interés.

Correspondencia:

Carlos J. Toro Huamanchumo

Dirección: El Muelle Ltes 5, 6 y 7. Dpto. 104. Las Delicias, Chiclayo, Perú.

Teléfono: +51 944942888

Correo electrónico: toro2993@gmail.com 